\title{
Discussion of "The New Role of Biomedical Informatics in the Age of Digital Medicine"
}

N. Al-Shorbaji ${ }^{1}$, R. Bellazzi ${ }^{2}$, F. G. B. de Quiros $^{3}$, S. Koch ${ }^{4}$, C. A. Kulikowski ${ }^{5}$, N. Lovell ${ }^{6}$, V. Maojo ${ }^{7}$, H. A. Park $^{8}$, F. Sanz ${ }^{9}$, I. N. Sarkar ${ }^{10}$, H. Tanaka $^{11}$

1

${ }^{2}$ Department of Electrical, Computer and Biomedical Engineering, University of Pavia, Pavia, Italy

${ }^{3}$ Direccion Medica, Hospital Italiano de Buenos Aires, Buenos Aires, Argentina

${ }^{4}$ Department of Learning, Informatics, Management and Ethics, Health Informatics Centre, Karolinska Institutet, Stockholm, Sweden

${ }^{5}$ Department of Computer Science, The State University of New Jersey Rutgers, New Brunswick, New Jersey, USA

${ }^{6}$ Faculty of Engineering, The University of New South Wales, Sydney, Australia

${ }^{7}$ Departamento de Inteligencia Artificial, Facultad de Informática, Universidad Politécnica de Madrid, Madrid, Spain

${ }^{8}$ College of Nursing \& Systems Biomedical Informatics Research Center, Seoul National University, Seoul, Korea

${ }^{9}$ Research Programme on Biomedical Informatics (GRIB), Hospital del Mar Medical Research Institute (IMIM), Universitat Pompeu Fabra, Barcelona, Spain

${ }^{10}$ Center for Biomedical Informatics, Brown University, Providence, Rhode Island, USA

${ }^{11}$ University Center for Information Medicine, Tokyo Medical and Dental University, Tokyo, Japan 


\section{Summary}

This article is part of a For-Discussion-Section of Methods of Information in Medicine about the paper "The New Role of Biomedical Informatics in the Age of Digital Medicine"written by Fernando J. Martin-Sanchez and Guillermo H. Lopez-Campos [1]. It is introduced by an editorial. This article contains the combined commentaries invited to independently comment on the paper of Martin-Sanchez and Lopez-Campos. In subsequent issues the discussion can continue through letters to the editor.

\section{Keywords}

Biomedical informatics, digital medicine, participatory medicine, precision medicine, social media, self-quantification, exposome

With these comments on the paper "The New Role of Biomedical Informatics in the Age of Digital Medicine" written by Fernando J. Martin-Sanchez and Guillermo H. Lopez Campos [1], the journal seeks to stimulate a broad discussion on the future role of biomedical and health informatics as scientific discipline. An international group of experts has been invited by the editor of Methods to comment on this paper. Each of the invited commentaries forms one section of this paper. 


\section{Comment by N. Al-Shorbaji}

Commenting on a piece like this one [1] is not an easy task especially that it was authored by two distinguished biomedical informaticians. They have expressed serious concerns about the discipline and even the profession itself but also made a clear way forward by stating that "the most decisive factor of progress is the proper use of data, information, knowledge and evidence." This confirms what we have been saying about health being a data intensive and knowledge-based sector [2]. The future of healthcare will depend on the quality and quantity of health data collected, analyzed and used for the benefit of the individual. The population in general and patients in particular will be better served by moving data about them rather than moving them to where healthcare services are located.The extreme concern is expressed at the end of the article when the authors say "These new, and not so new, domains are becoming a real threat, which could rip and tear our discipline, stripping it away from some of our most relevant areas of work". They concluded by saying "If Biomedical Informatics does not start to significantly embrace and complement the emergence of these new disciplines, we could be in grave danger of becoming irrelevant. The cost of this to our patients and our society could be devastating". This reflects a very dark view of how disciplines interrelate. It projects a view of a single-handed branch of science attempting to do everything for humanity. To explain this point further, and despite the fact that the article is loaded with terms and has a rich discussion on terminology, it did not cite any of the systematic reviews of eHealth terminology (by accident or on purpose) [3] [4] [5]. The denial of the existence of this body of knowledge did not help in mapping a future direction for the profession or the discipline.

With the diversity of definitions and descriptions, it has become clear that we are all talking about the same thing which is "the use of information and communication technology in health". The description of eHealth provided by the World Health Assembly resolution of 2005 [6] still stands as it says "eHealth is the cost-effective and secure use of information and communications technologies in support of health and health-related fields, including healthcare services, health surveillance, health literature, and health education, knowledge and research".

The core concept for which the authors are making the case is understandably "Biomedical Informatics" and "biomedical informaticians". The authors are confident that "informaticians" can do and should do a huge list of tasks. I, for sure, do not agree that the "informaticians" have an infinite capacity, expertise, knowledge and skills to do all this solo, as implied in the article. They need to be more humble by being more open to working with other professionals to form teams that can better understand and deliver programmes and produce research on areas such health promotion, standardization and interoperability, system design, clinical trials, digital imaging, data analysis, etc.

The space of eHealth is wide enough and should be fertile enough to absorb professionals from multiple disciplines and then blend them to help in creating a good health service delivery system that is comprehensive, accessible, universal, equitable, safe, cost effective, 
secure, sustainable and accountable. Such a system cannot be designed and managed only by "informaticians".

Two additional complexities added in this paper: one is in its title as it refers to "age of digital medicine" and we all know for fact that "health" is much bigger than "medicine" which has been fully recognized in the paper itself when referring to the different aspects that contribute to the health of people including the environment and other elements. The other one is the term "informatician" which is not recognized even in language dictionaries. These dictionaries refer always to "informatics" and the closest to it being Bioinformatics defined by Oxford Dictionary [7] as "The science of collecting and analysing complex biological data such as genetic codes." The paper itself does not offer a comprehensive definition of biomedical informatics.

Hersh [8] in 2006 asked the question "Who are the informaticians?" and said "By these definitions, informatics has some characteristics of a profession. While there is a continued growth in the self-identification of medical informaticians, the field still lacks a formally defined curriculum and set of agreed-on competencies. Some analyses have assessed the curricula of educational programs and how they match emerging definitions of professional competencies, finding a great diversity in curricula".

The challenging question is "is biomedical informatics" a discipline and that informticians are the professionals within this discipline or it is just a self-identification issue?" This might be the case for all the other self-identified groups and disciplines that the paper excellently referred to in different countries and in different settings.

I am, therefore of the opinion to use the term eHealth as an umbrella term to refer to all the other old and emerging disciplines and concepts. I would not go to the extent of creating a new term such as "eHealthian" or "eHealth Scientist" or "Digital Health Scientist" taking "Big Data Scientist" as a model. I am of the opinion also that one day the "e", the "tele", the digital, and the " $u$ " etc. will disappear and we will continue to use health and medicine especially that computers (i.e. digital data processing and communication equipment) will be in every single discipline and human activity. What we have been observing is emerging terminologies describing activities that focus on "the most decisive factor of progress (which) is the proper use of data, information, knowledge and evidence" as the authors of the paper said on page 9 . We have been observing healthcare professionals getting interested in the use of ICT in health and/or ICT professionals finding themselves in the middle of a health project in which ICT is heavily used. In both cases the two professions learn from each other's and these professionals become a new hybrid breed.

Therefore, I would like in the rest of my comment to focus on two issues that I feel of great importance as a contribution to this discussion:

\section{1. eHealth is multidisciplinary/interdisciplinary}

The article never made a reference to biomedical informatics or any of the disciplines that it discussed as interdisciplinary or multidisciplinary. It referred to the "intersection" between 
certain disciplines. The Free Dictionary on the Internet defines interdisciplinary [9] as "it involves the combining of two or more academic disciplines into one activity (e.g. a research project). It is about creating something new by crossing boundaries, and thinking across them". The Dictionary even used bioinformatics as an example to demonstrate the meaning when it said "Bioinformatics as an interdisciplinary field combining molecular biology with computer science". [10]

Different reviews have made the point clear that within the practice of eHealth or any of the alternative terms and concepts used to describe the healthcare delivery through the use of information and communication technology (ICT) one has to establish one form or the other of a multidisciplinary/interdisciplinary team. That team has to have ICT professionals who know computer hardware and software and who know telecommunication infrastructure, methods and protocols. The team has to have healthcare professionals being nurses, clinicians, pathologists, radiologists or any other health-related professional. That is probably why we have the term "tele-medicine" and all the other medical or health specialties which created terms such as tele-radiology, tele-psychology, tele-... etc. If it is not about team formation, it is then about formation, education and training of the eHealth professionals. That professional has to be specialized in a health-related field and conversant with ICT or vice versa. One cannot have a radiologist or a pediatrician as biomedical Informatician without proper training in the use of ICT. The power of combing the two disciplines is what makes this new discipline unique and worth what it is worth. Billions of dollars are spent on eHealth projects, patient safety is at stake as a result of using or not properly using ICT in healthcare, a huge body of research has emerged on the evidence and value of use of ICT for health and a huge number of professionals are practicing ICT for health in all healthcare units. Bahgon and Jacobson [11] concluded that "Successful implementation of eHealth solutions is not a sum of technology, innovation and health; Rather it's the expertise of tailoring knowledge and leadership capabilities in multidisciplinary areas: clinical, ethical, psychological, legal, comprehension of patient and medical team engagement etc." Van Velsen, Wentzel and Van Gemert-Pijnen [12] went further to say "The potential of eHealth technology can only be fully exploited when it is developed by a multi-disciplinary team who apply a human-centered approach that takes the specifics of the context (both organizational and that of the individual user) in which the technology is to be used into account".

\section{Universal Health Coverage in the Sustainable Development Goals}

At the UN Conference on Sustainable Development (Rio+20), held in Rio de Janeiro in June 2012, 192 UN member states agreed to establish an intergovernmental working group to design the Sustainable Development Goals (SDGs) as a successor of the Millennium Development Goals ( MDGs) [13]. The Sustainable Development Goals (SDGs), officially known as transforming our world: the 2030 Agenda for Sustainable Development, are an intergovernmental set of 17 aspirational Goals with 169 targets. The SDGs were approved by the United Nations General Assembly in September 2015. [14] 
The SDGs are very broad and complex in general as they address all aspects of human development. Health, which is our concern here, is in a relatively good position as SDGs provide a very comprehensive set of health targets with multiple linkages to other SDGs as contributor and beneficiary. The SDGs represent a set of "Integrated and indivisible" targets and progress in one area is dependent on progress in many others. This simply means achieving an SDG will automatically contribute to the achievement of another. Improving education, elevating poverty and improving housing conditions, etc. will automatically help better health status of the individual and the community at large.

Now considering the terminology used by Sanchez1 and Lopez-Campos in the paper which includes terms like "exposome" which was coined by Christopher Wild [15] (currently Director of the WHO IARC in Lyon) in his 2005 article saying "At its most complete, the exposome encompasses life-course environmental exposure (including lifestyle factors), from the prenatal period onwards."

Health in the SDGs is represented as Goal 3 which reads "Ensure healthy lives and promote well-being for all. In addition to Goal 3 on health, health is heavily represented either to contribute or to benefit from other goals and targets such as ending poverty, equitable education, gender equality, ensure availability and sustainable management of water and sanitation for all, end hunger, achieve food security and improved nutrition and promote peaceful and inclusive societies for sustainable development. The Goal itself has 12 targets distributed in three groups as follows:

1. Targets on the unfinished and expanded MDGs:

- 3.1: Reduce maternal mortality

- 3.2: End preventable newborn and child deaths

- 3.3: End the epidemics of HIV, TB, malaria and NTD and combat hepatitis, waterborne and other communicable diseases

- 3.7: Ensure universal access to sexual and reproductive health-care services

2. New MDG Targets:

- 3.4: Reduce mortality from NCD and promote mental health

- 3.5: Strengthen prevention and treatment of substance abuse

- 3.6: Halve global deaths and injuries from road traffic accidents

- 3.9: Reduce deaths and illnesses from hazardous chemicals and air, water and soil pollution and contamination

3. Means of implementation target:

- 3.a: Strengthen implementation of framework convention on tobacco control 
- 3.b: Provide access to medicines and vaccines for all, support R\&D of vaccines and medicines for all

- 3.c: Increase health financing and health workforce in developing countries

- 3.d: Strengthen capacity for early warning, risk reduction and management of health risk.

Target 3.7 on Universal Health Coverage is comprehensive and may stand on its own as inclusive to all others. It considers all aspects of health care delivery. The Target reads "By 2030, ensure universal access to sexual and reproductive health-care services, including for family planning, information and education, and the integration of reproductive health into national strategies and programmes". WHO [16] stated that "The goal of universal health coverage is to ensure that all people obtain the health services they need- prevention, promotion, treatment, rehabilitation and palliation without risk of financial ruin or impoverishment, now and in the future. Prior to that and based on the WHO report of 2010 [17], UHC was adopted by the United Nations General Assembly resolution in 2012. [18] Universal Health Coverage (UHC) provides a platform for an integrated approach for the SDG health-related targets that should overcome the compartmentalized approaches of the previous MDGs. The SDG agenda provides a critical opportunity to consider health in a much more integral manner with the economic, social and environmental goals.

With the language used in the Sustainable Development Goals and more specific in the UHC we find great synergy between them. The health of the individual is not only a result of gene sequence, phenome or exposome. Health is the result of all these. The language that is used by scientists, including biomedical informaticians and the language used by politicians and health economists are different despite the fact that they mean the same thing.

The proper use of data, information, knowledge and evidence is the common denominator between what biomedical informaticians want to achieve and what politicians want to achieve. SDGs are driven by data and science and can only be achieved if data, information, knowledge and evidence are properly used.

In conclusion, there is a huge opportunity for integration of healthcare and medical services through data and there is huge opportunity for interdisciplinary team work both in the academic world, research and practice. This opportunity should not be lost as a result of emerging terminologies and professions on the expense of target populations being healthy or sick. Health services should not only target the sick people, more important as stipulated in the paper to go for the prevention, protection, promotion, treatment, rehabilitation and palliation. This is about care during the whole life of the individual leaving room for professionals form all disciplines to use ICT to manage health data in proper and ethical manner. 


\section{Comment by R. Bellazzi}

Biomedical informatics (BMI) is a fascinating discipline that stands at the intersection of many fields, from computer science and informatics to medicine and biology. Its ultimate goal is supporting biomedical decision-making and scientific discovery by the proper use of data, information and knowledge [19]. BMI has, by nature, soft boundaries, and its core competencies (https://www.amia.org/biomedical-informatics-core-competencies) are general enough to be expanded and revised when needed.

In fact, BMI has a peculiar position: it is instrumental to achieve goals of other disciplines, such as clinical medicine and translational biomedical sciences, it relies on results of other disciplines, such as statistics and social sciences, and it applies technologies coming from other disciplines, such as computer science and bioengineering. In other words, BMI can be considered as the bridging discipline of biomedical information [20]. For this reason, it is probably more engineering than science, thus playing a crucial role in the effective implementation of biomedical information systems. However, as it happens with engineering, it produces original scientific and technological results that contribute to the growth of the body of fundamental knowledge.

The paper "The New Role of Biomedical Informatics in the Age of Digital Medicine", by Fernando Martin-Sanchez and Guillermo Lopez-Campos [1], focuses the attention of the BMI community on the concurrence of three important drivers of change that are nowadays impacting on medicine: participatory health, precision medicine and big data science. Also due to such factors, medicine is (finally) moving fast towards the digital world [21], so fast that NIH has a specific goal to "build a digital ecosystem for biomedicine" [22] (datascience.nih.gov), and that many IT industrial players are increasingly involved in large scale biomedical projects.

The paper stresses that, paradoxically, BMI run the risk of becoming marginal in its own field, due to the concurrent combination of two factors:

i) the tendency of researchers and practitioners of staying in their perceived comfort zone, such, in the BMI case, electronic health records, biomedical terminologies and standards, statistical analysis of clinical data; this may lead to miss challenges and opportunities posed by precision medicine [23, 24], participatory health [25] and exposomics [26].

ii) the uneven positioning of the national medical informatics societies in the different countries, both in terms of their scientific standing and of their impact on national policies.

These two factors may open space to initiatives that will soon start to rediscover the wheel, with the obvious effect of wasting time and resources of the research community and, at the same time, of posing a serious threat to BMI as a field.

The paper is properly highlighting that the current period is characterized by a lucky combination of technology push (big data technologies) [27] and market pull (precision medicine) [28]. It is therefore essential to be able to exploit such combination by making BMI as the "epicenter" discipline for biomedical information, following the NIH/NLM approach. 
This changing role of BMI will require to re-use and only to partially transform core competences that belong to our community, such as data representation, data integration, information extraction, knowledge engineering, data analysis and mining.

As previously mentioned, BMI has an engineering nature and a problem-solving inspiration, it has soft boundaries and it may naturally embrace a number of relevant technological and methodological challenges, including [29]:

- To be able to deal with the participatory medicine "revolution", adding strategies to manage, represent and distillate information coming from patients;

- To include exposomics in the holistic scenario of managing information that goes from molecules to population.

- To leverage on the ability of handling data variety by integrating and fusing biomedical data, images, signals, text to support decision-making.

- To build, managing and analyzing very large databases and distributed data resources.

- To support translational bioinformatics in the process of feeding precision medicine with results coming from fundamental molecular and biological knowledge

- To exploit big data technologies and to implement big-data ready computational frameworks

In a nutshell, to cope with the risk of staying in the comfort zone, BMI will have to continue to do (and not to forget) what it has done over the last 50 years, by continuously adapting to problems posed by the society and by exploiting the opportunities offered by emerging technologies. Grounding on the fundamental relationships between data, information and knowledge, BMI will have to plug and play new data, new technologies and new problems to providing a growing impact to the scientific community and to the people. This is of course particularly challenging, especially in educational programs, since education is necessarily slower than technology [30, 31].

Moreover, in the future, BMI will need to establish cross-fields alliances, in particular with the other scientific communities involved in the biomedical informatics game. For example, the coordination with BMI groups and conferences in the area computer science and bioengineering is crucial for cultural and strategic reasons. As already previously mentioned, participatory medicine and big data requires constant injection of advanced methodological contributions in the area of signal processing, very large databases, networking and distributed AI. Furthermore, it will be important to reinforce existing links with translational bioinformatics and computational genomics, to strengthen relationships with public health and to start specific initiatives in environmental health.

As concerns the second remark of the paper by Martin and Lopez-Campos about the weakness of BMI societies and BMI perception in some key countries, it is easy to note that this is a real potential limitation for the growth of the field. Being BMI a discipline that is 
strongly interrelated to other ones, it runs the risk of being subsumed. In many countries, biomedical researchers and decision-makers do not even consider BMI as a separate discipline that require specific know-how and formal training. This impacts on the decisionmaking process, which very often is in the hands of managers without any BMI education. The situation is obviously critical, because, the lack of BMI culture and recognition leads to under-estimate the complexity of informatics interventions, with negative consequences on costs and outcomes.

The weak positioning of BMI has other negative impacts: for example, BMI has not been included in the list of disciplines explicitly considered by the panels of the European research Council (ERC). The ERC panel structure consists of "25 panels, grouped in three disciplinary domains, covering the entire spectrum of science, engineering and scholarship in the remit of the ERC: Social Sciences and Humanities, Life Sciences, Physical Sciences and Engineering" (https://erc.europa.eu/evaluation-panels). The lack of BMI (while bioinformatics is in the list) makes it very hard for BMI researchers to apply for ERC grants. Moreover, since the ERC panels have been considered as the starting points to classify grants application in some EU countries, like Italy, the same problem occurs downwards.

In Italy, by the way, the situation of BMI is quite critical. BMI has been scattered in different groups and society belonging to different cultural provenance (physicians, engineers, bioinformaticians), and, at the moment, there is no single society affiliated to IMIA. Needless to say, previous attempts have been poorly able to contribute to the digital health efforts of the country. For this reason, the Italian Biomedical Informatics Society (SIBIM) has been created this year, with the hope to gain a scientific leadership and visibility that may provide a reference for the BMI national initiatives.

However, only a joint and coordinated action of international and national societies and federations can be truly effective.

As a consequence, it is becoming urgent that BMI international organizations, such as IMIA and EFMI, devote consistent efforts in reinforcing the role of BMI in the policy-making processes, and in supporting national societies to increase their local influence. The role of the most successful national associations is of paramount importance, too, in order to strengthen the message that BMI academic training and research is mandatory in the era of digital medicine. AMIA, for example, has been successful to influence precision medicine policies and governmental actions. Its support and example can be beneficial also for other countries.

Rephrasing the last sentence of Martin-Sanchez and Lopez-Campos paper, an "inconvenient truth" is that BMI, much more than other fields, cannot rest or slow/down, but rather, it must keep running fast to be able to continue to contribute to health care, science and, finally to people lives. It is our responsibility, as researchers in the area, to find the balance between the need of stabilizing BMI as a discipline, strengthening its standing and visibility, and, at the same time, of maintaining its dynamic and open nature. 


\section{Comment by F. G. B. de Quiros}

Digital medicine will be finally driven by patients or physician needs?

The article by Martin Sanchez and Lopez Campos [1] describes the concept of Digital Medicine, how precision and participatory medicine play an important role in this scenario, and the new "role" that the patient has in this context with the use of digital technology in the everyday life [32]. We can say that the concept and principles of precision medicine are not new since it is one of the main objectives of the medical practice to classify diseases or conditions the patient has and work on how to prescribe a specific treatment based on a diagnosis. What have changed are how new advances in diagnostic and treatment options are available adding social and environmental factors that enhance and open a great amount of options that make it hard to integrate without the help of information technology, because of the convergence of genetic and molecular information. This poses a new dilemma on how to properly manage the right treatment for the patient [33].

Interest in precision medicine is shared, but different points of view and needs, from patients, physicians, health systems, payers, and the diagnostics and pharmaceutical industries that may not be completed aligned.

The patient that has been using IT tools in their daily life, when a condition or disease is present they will try to look for a better understanding of their disease, including the best and effective therapy available, together with the prognosis. It can be a burden for the healthcare system (including physicians) to articulate and find a proper balance between the patients' interest and needs with the sensitive use of resources, since most of the new diagnostics tests and treatment options have risen the costs of healthcare making it in some situations difficult to be instrumented in many developing countries, since it is home to $84 \%$ of the world's population, but only accounts $12 \%$ of the global spending on health. On the other hand in developed economies the costs of healthcare have risen to a point that the system might collapse. So there is a risk that precision medicine might only be a privilege for those who can "pay for it", adding another item to the big list of health disparities that developing countries are dealing with [34].

As we have said earlier in this new context of digital medicine the patient plays a new and important role driven by the use of IT, this has been explained in Topol's "Creative Destruction of Medicine" where he describes how medicine is changing the approach of treating medical conditions from the traditional population-based methodology to individualized or personalized medicine with the use of digital technologies that work as driving forces of this transformation. Topol proposes that healthcare can improve when patients are involved in the decision on what treatment to have, when they have more information that is properly used and provided. Nowadays people rely on social media and any kind of information available on the Web for making health decisions so the current challenge is how to help them in getting the right information that might not lead to inappropriate or unnecessary use of healthcare procedures [35].

It is fact that patients are being gradually more involved in Social Media [36] so the healthcare team needs to address this issue and try to establish social network intervention 
strategies: first try to identify and promote champions or opinion leaders as agents of change; also try to identify groups in order to stratify or what kind of people need to be addressed; find ways of stimulating the "viral effect" by peer to peer sharing of information and finally try to be part of the social network by participating and introducing health advisors and try to engage other groups, agencies or NGOs to work on a common purpose. [37]

There is new scenario and it's changed the traditional form of care driven by the active involvement of patients, this results in a medical care that has become more "population based", since patients are driving their own care, lets hope that this new situation can help the development of digital medicine. 


\section{Comment by S. Koch}

Healthcare is considered lagging behind other industries regarding modern information technology [38], especially mobile technology [39] and healthcare professionals often use more advanced technologies in their private life than they are offered at their workplace. Healthcare managers seek for ways to speed up digitalisation in hospitals and to manage clinical workflows more efficiently through information technology. It is not new that such situations call upon technology-driven initiatives. Such initiatives generally have in common that they ignore Biomedical Informatics as a discipline. In this context, Martin-Sanchez and Lopez-Campos discuss the implications of the arising field of Digital Medicine on Biomedical Informatics [1]. Defining Digital Medicine as "the convergence between the digital revolution and medicine" [1], they emphasise new research opportunities for biomedical informatics along two lines, namely "participatory and precision medicine". To solve the informational problems in these two areas by adequately managing genome, exposome and phenome data as well as their interactions is considered to be at the core of Biomedical Informatics. This should also be considered the advantage of our field in respect to Digital Medicine.

\section{Competition between hype and science?}

Martin-Sanchez and Lopez-Campos visualise the increasing popularity of Digital Health compared to Biomedical Informatics through a Google Trends search [1]. Replicating the same search in scientific databases (namely PubMed and IEEE Xplore) renders another image. Biomedical Informatics is mentioned 20 times more often than Digital Health in PubMed and 10 times more often in IEEE Xplore and a decreasing trend cannot be identified yet. This does however not lessen the problem of new tech terms appearing and threatening our field. About 15 years ago it was the term eHealth that appeared [40] and that is now on the way to disappear and be replaced by Digital Health. We have the advantage of a comprehensive view on an integrated discipline and we need to focus on the scientific basis of our field. However, we also need to get better in promoting our discipline to those outside the biomedical informatics community. An inherent problem of the field of Biomedical Informatics is that Informatics as a discipline is interpreted differently in different countries and that we have so many ways entitling it [41]. We combine theories, methods and models from many fields such as information systems, computer science, cognitive science, biology and human-computer interaction apart from developing our own [42], which is, both a strength and a weakness. A strength, because we grasp the field of biomedical informatics in a holistic way and a weakness because we struggle to systematically describe our own theories, methods and models and a scientific description of biomedical informatics from an informational perspective is still lacking [43]. Without this, we will have difficulties moving science forward and promoting our field. 
Towards preventative medicine

Martin-Sanchez and Lopez-Campos regard precision and participatory medicine as contributing ideas towards the more ideal concept of preventative medicine [1]. Precision and participatory medicine are complementary, both regarding types and content of data. Whereas we have been collecting data for precision medicine structured and systematically, participatory medicine is still rather unexplored from an informatics point-of-view. Patients take control over their health and Martin-Sanchez and Lopez-Campos consider the electronic health record to be one of the best tools to do so. However, we know that even tethered personal health records do not fulfil the requirements patients have towards them [44] and previous studies reported that computerized history taking by patients outperformed routine care in collecting historical data relevant for risk stratification [45]. Patients will provide us with new types of data that will be of relevance to achieve preventative medicine. Social media, mobile health apps, and self-tracking devices enable patients with chronic diseases to track their health or to achieve a specific aim, e.g. adjustment of medication [46]. Patients engaged in the quantified-self movement [47] increasingly set-up their own single subject design studies (n-of-1 trials). Biomedical informatics offers the methods and tools for the design and analysis of (series of) n-of-1 trials making them accessible to clinicians and patients and supporting the seamless integration of n-of- 1 trials into clinicians' practices and patients' lives [48]. This area will not only provide exciting challenges for biomedical informatics but also enhance systematic data collection on the comparative effectiveness of treatments directly in the practice setting.

In conclusion, I agree with Martin-Sanchez and Lopez-Campos in that the era of Digital Medicine provides new, exciting opportunities for biomedical informaticians and we need to make sure we are on the driving seat of this development. 


\section{Comment by C. A. Kulikowski}

This paper [1] elaborates on a keynote presentation by the first author at MIE 2015 in Madrid. It discusses the challenges, risks and opportunities presented to BMI by the increasing penetration of digital devices, their computational power, and rapid, mobile communication capabilities, which are not only affecting the practice of medicine and health care in general, but also affording more direct engagement of patients and consumers with their practitioners, raising difficult issues for personalization in "digital medicine". The authors also address challenges involved in biomedical research in terms of the promises of "precision medicine" which will require considerable advances in the underlying sciences and their ability to help us understand the "translations" of genomic and other basic science discoveries into comparable discoveries about what might be suitably useful clinical phenotypes based on genotypical, developmental, behavioral, and environmental information.

The paper offers a comprehensive overview of the definitions of digital medicine, its evolution, and how BMI has, and is likely to affect its practice. It is very up-to-date with current nomenclature of the myriad ways in which informatics contributes to, and is affected by, developments in Precision Medicine - such as the exposome concept - and Participatory Health - such as the Quantified Self projects.

As in any broad position paper, there are many aspects one can question and disagree with. In Table 1 the authors compare and contrast the differences between Personalized and Precision medicine, some of which might be generally conventionally accepted, but others not so, with unnecessarily constraining results for the arguments made. These include, under the criterion of Data Sources, that personalized medicine focuses on just genomic and clinical data whereas Precision Medicine is multidimensional, by adding molecular, multi-omic and other exposome data sources. This choice of contrast can be disputed as simply being an artifact of the early stages of both fields, where Precision medicine has yet to produce enough multiomic and exposomic results to be widely recognized as useful to most practitioners for routine clinical application. Likewise, for their criterion of Patient Monitoring where the contrast given is between discrete and static "snapshots" of a patient's condition for Personalized Medicine vs Precision medicine looking for the best continuous, dynamic stratification for modeling a patient's clinical journey. This can be seen as a distinction between the largely scientific, research oriented analysis of population data in the latter vs. the individual clinical goals of the former - also a reflection of the early stage of precision medicine and efforts at personalization. One can argue strongly against this contrast, since producing a static "snapshot" view of a patient during a clinical visit is usually due to operational factors of healthcare: limited time and the considerable effort needed to systematically update the full clinical history record of a patient being monitored. In the "good old days" of clinical medicine where a physician or nurse spent as much time as needed to listen to the patient and his/her family, and kept meticulous written records of what was said as well as what the physical examination showed, the dynamic long-term temporal analysis modeling the patient's journey was done much more comprehensively, though maybe not with the 
"precision" that the new label for this kind of medicine pretends to in incorporating -omic and other exposomic data (presumably precise, but rarely yet so!).

The contrasts chosen for the last two categories given in Table 1 are likewise debatable: personalized medicine is only reductionist for practitioners who are themselves reductionist and seeking easy and quick answers under the pressure of time - good clinicians are equally integrative and "system thinkers" as anyone who is working in precision medicine. One could argue that precision medicine will not go very far unless it works with such clinically insightful and caring physicians. For the final criterion of patient role, listing it as passive for personalized medicine is more a function of the social mores and "positivistic science" approaches of medicine of the 19th and 20th centuries - authoritative opinions on the part of physicians and nurses, and definite "evidence-based" rules for diagnosis and therapy as the expected outcomes of clinical research. This ignores developments of the past 30-40 years, where increasing insights from behavioral, cognitive and social science are beginning to influence scientific thinking in more nuanced ways, while having some impact on healthcare practitioners in moving them away from traditional authoritarian behavioral models. This commentator finds such sharp and rather simple contrasts not as useful as they might have been with a deeper analysis.

The third section of the paper concludes with a discussion of participatory medicine which emphasizes efforts that involve patients in their self-care through various technological and informatics means. The authors use it to highlight the central role of informatics in these efforts, which is well-taken.

Martin-Sanchez and Lopez-Campos then proceed to give examples from their own experience that point to the challenges, opportunities and dangers for informatics in the move to mobile, personalized medicine. They describe the results of a survey they carried out on what is described as the "affordances" of social media (self-presentation, connection, exploration, narration, and adaptation), which influence how people use a particular artifact in particular ways. Their conclusion is that while technological platforms change often, modalities of patient interaction are always relevant and affordance factors correlate with health outcomes for chronically ill patients - not a surprising result. They then move to describe a QuantifiedSelf project which they worked on in Australia where they investigated the information flows from a self-monitoring device to a smartphone, to the cloud, and looked at different information architectures and communication protocols involved. They published a white paper with a proposed classification scheme relating self-quantifying devices and software to human activities related to health - sleep and exercise. This is presented as a first attempt to help develop minimum information standards for the description and annotation of experiments in this field.

The authors then provide a brief description of their notion of exposome informatics, pointing out how it fits at the intersection between participatory and precision medicine, pointing to the needs and virtues of collaboration between biomedical-health informatics and bioinformatics. This is not a new plea, but one that typically is not realized in practice due to many divergences as well as overlaps in goals, background, education and self-selection in these 
fields as discussed in [49]. In the next section on the changing role of biomedical informatics the authors emphasize that the earlier layered molecule-to-population view of biomedical informatics [50] usually neglected environmental factors as emphasized by the exposome and expotype projects, pointing out how massive amounts of individual data can now be gathered from patient/subject specific devices over the lifetime of a patient. They then show an information flow diagram to describe these processes.

The final section of the paper entitled Perspectives describe some of the professional experiences of the authors as members of the biomedical informatics communities in Australia and Spain, noting the differences in history and coverage of topics, which in both cases focused on health informatics and its applications, and paid little attention to foundational research in BMI. For Spain they mention that two new associations dealing with Digital Health and eHealth have been formed, challenging the existing IMIA-affiliated SEIS organization. The authors question whether societies that are centered more on the technology, communication and business aspects rather than on the core of scientific informatics issues of standards and interoperability and usability of systems can really work without well-trained biomedical informaticians. They then go on to argue that it is informatics alone which is ready to tackle the complex issues of empowering patients in the use of new informatics technologies in this age of digital medicine, starting with the electronic health record as a means by which patients can be allowed "more control over their own health" taking technological device integration, cognitive approaches to decision support, while also contributing to evolving developments in data analytics, visualization and presentation. They argue for the need for n-of-1 or single-subject clinical trials, which raises scientific, mathematical modeling, and bioethical issues - but which the authors do not identify specifically.

In summary, as an overview paper that looks into the future of biomedical informatics as a unique field for bringing a scientific approach to biomedical problems of human-technology interactions, the paper touches on many of the relevant points in a broad and easy-to-read manner.

The paper unfortunately does not provide a satisfactory sense of where the field finds underlying informatics "grand challenges" - such as those involving the integration of different ontologies, that are sufficiently fundamental and deep themselves that they do not yield to the easy re-labeling of existing mathematical, statistical, and computational models and methods or description by superficial and largely content-free analogies captured by ambiguous conceptual graphs. The paper also neglects to go into the complex factors that can account for the harms rather than benefits, which technology can introduce into healthcare practices [51]. Asking biomedical informatics to embrace a number of newly labeled fields such as participatory or precision medicine is reasonable, but as stated above, the rationales given are not always the most convincing. So, this is a thought-provoking paper on possible future connections of biomedical informatics to digital medicine, even if short on new insights into how these connections may play out. 



\section{Comment by N. Lovell}

\section{What's in a name?}

Martin-Sanchez and Lopez-Campos in their paper "The new role of biomedical informatics in the age of digital medicine" explore the role of biomedical informatics (BMI) in not just facilitating progress in research fields that are the foundation of digital medicine, but also in supporting the practice and delivery of health care. They expound on the evolving and converging fields of information technology and health care, noting the identity crisis that is potentially facing the BMI profession. This identify crisis, while more relevant today due to the way ideas, thoughts and concepts can be widely adopted and promulgated, has faced the field for many decades. The authors note that public health informatics applies at the population level but in certain areas of the world the concept of health informatics and BMI are used loosely and nearly interchangeably and span levels of hierarchical scale. They report on the popularity of a number of key terms by way of Google Trends, showing that BMI has afforded relative stability over the past decade, while other terms have waxed and waned. While the authors themselves did not explore other informatics terms, on conducting a similar search in Google Trends we see that the term 'health informatics' has also been stable over the last five years and being used some four times more frequently in searches when compared to BMI. On the other hand 'bioinformatics' was hugely popular a decade ago (some 100 times more trending than BMI) but has gradually declined to be about ten times more used than BMI today.

The reality is that we live in a society that is based around reinvention, with people and groups wishing to add their own contribution to the field, not to mention companies promoting technologies and associated standards in order to obtain competitive edge. One means of so doing is to use branding. Fields and areas of research may not change dramatically but they morph as they adopt the cloak of a new name. With our connected world, the morphing process is becoming easier but the BMI profession needs to recognize this fact and adopt to the introduction of new terminology and branding.

\section{The medium is the message}

The popular social commentator of previous generations, Marshall McLuhan, back in 1966, said that "the medium is the message". He wrote that various communications mediums have distinct impacts on the consumers of media. While McLuhan died well before the digital age, not to see the rise of social media and pervasive information and communication technology (ICT), his vision extends to the current, with BMI not just a tool for digital medicine but indeed shaping the way healthcare is both delivered and practiced. Current healthcare delivery is shaped in myriad ways by ICT, with the abundance of technology and access to information being the driver, for example, for the rise of participatory medicine.

Perhaps the next wave of change that the authors did not specifically address is that of 'The Internet of Things' (IoT). The analyst firm Gartner says that by 2020 there will be over 26 billion connected devices [52] and Forbes has estimated that the healthcare market for IoT will reach US $\$ 117$ billion by the same year [53]. With wearable, implantable and point-of- 
care technologies all having the capability to report on not just one's physiological measures but also the way a person interacts with their environment and possibly changes behavior in the onset of illness, the author's emphasis on the Exposome becomes even more important and significant.

\section{Plentiful preponderance of ' $P$ ' prefixes}

The authors discuss the concept of Personalized, Precision and Participatory medicine. But the ' $\mathrm{P}$ ' fest as a medicinal adjective does not stop there. Zhang and Poon [54] in their 2010 editorial in the IEEE Transactions on Information Technology in Biomedicine (now renamed the Journal of Biomedical and Health Informatics), extended this to a 6-P 'p-Health' paradigm, highlighting 3-Ps for what kind of healthcare decisions should be made (in a Preventive, Pre-emptive and Predictive fashion), and another 3-Ps for how healthcare decisions should be made (in a Personalized, Participatory and Pervasive manner).

Is there more alphabet soup when talking about BMI and a digital medicine nexus?

The genesis and development of participatory medicine in the age of ubiquitous social media, creates significant dilemmas for the profession, the industry and regulators alike. Citizens expect both the availability and connectivity of multiple data feeds, as well as data to be automatically and accurately synthesized into knowledge.

Martin-Sanchez and Lopez-Campos briefly mention interoperability but don't elaborate on a number of critical areas that have now arisen in the age of digital medicine. It would seem somewhat appropriate in this discussion to consider an analogy between the development of the field and an electrocardiogram. What follows from the origin of the heart contraction initiated by the $\mathrm{P}$ wave, is a QRS complex. In our analogy, the ' $\mathrm{P}$ ' letter represents the beginning of many aspects of this p-Health era of digital medicine as elaborated in the previous section. But what follows - the QRS complex - is the need for Qualified, Regulated

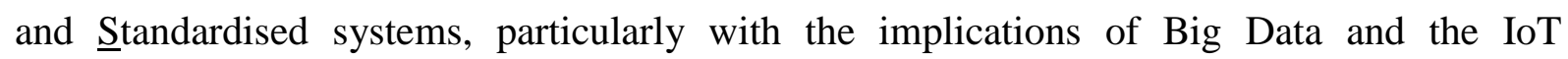
revolution. This uncertain situation has arisen for a number of reasons, including the complexity and enormity of managing qualification of the thousands of emerging smartphonebased products, the resistance and influence of multinational players, and even simple lack of awareness of the issues by many involved - particularly academicians.

Qualification of device efficacy and safety, regulatory approval of a medical device and adoption of various standards for safe device operation, accurate analysis, and device and communications interoperability are all interwoven. The complexity of regulatory approval is probably the greatest uncertainty moving forward. As an example, in February of 2015 the USA Food and Drug Administration (FDA) amended previous guidance on the regulation of medical mobile apps to try and instill some clarity for developers and manufacturers on whether their product required regulatory examination and approval as a medical device. In simple terms the distinction is primarily drawn based on whether a device, app or software is used in the clinical care loop or is a personal lifestyle/wellness application. The former requiring approval, while the latter does not. However it is safe to say that a large number of existing apps that are currently available providing patient-specific analysis, diagnosis or 
treatment recommendations are not compliant with the national regulatory authorities in the countries in which they are being used.

Riding the wave

Martin-Sanchez and Lopez-Campos place a timely call to the BMI profession to acknowledge a wave of change and to be ready to ride this wave. With IoT, pervasive communications and computing, and an increasingly connected world, being coupled with a desire and necessity to adopt aspects of the p-Health model, this wave of change is like none that have passed before - a true tsunami. Due to the 'QRS' issues outlined above and issues of privacy, security and ethical concerns, the environment is proving to be even more turbulent. The argument that the authors' make is that the BMI profession is perfectly placed to shape such advances, riding the wave of change. 


\section{Comment by V. Maojo}

\section{Proliferating Fields and New Terms around "Digital Medicine"}

I want to thank, first, the MIM editors for inviting me to participate in such an interesting debate. In this case, I believe that the authors aim to shoot at the right target by addressing the concept of "digital medicine", surrounded by other recent hot terms such as "digital health", "precision medicine", "participatory medicine", "health 2.0" and many more that arise in recent papers, the Web and social networks. All of them needed to be addressed in an extended paper, from a biomedical informatics (BMI) perspective, given their proliferation. Thus, in addition to being timely, based on the experience of the authors in the area, and being written in a very readable form, this paper is reasonably comprehensive in addressing a large number of issues linked to the current novel digital technologies around biomedical research and clinical practice. The use of data from multiples sources - e.g., environment, body sensors, omics, external devices, integrated datasets, etc - surely adds substantial information that will influence future healthcare approaches. However, the extended view of many current trends presented in the manuscript - more broad than deep —, can lead, similarly, to lack of a specific, concrete focus. It is difficult, in fact, not to fall into this subtle trap if one wants to address the many topics that one can observe in what is called "digital medicine". Finally, like the authors, when they state that nowadays patients are no longer patient, one can also finish reading the conclusions of the paper feeling that there is not a clear conclusion.

As well-pointed out by the authors, the original idea of the "e-patient" - including electronic sources of information and devices for healthcare- has been around for a long time, but what has dramatically changed is the perception of laypeople, the media and particularly many companies - both large and small - that realize now their increasing opportunities of doing a lucrative business in healthcare information. In this new social and media context, it is not strange that other previous, recent though incomplete accounts of what this "new" digital medicine is, have been largely successful in terms of readership, despite lacking an accurate scientific analysis. By way of example, a recent best-seller book, "The digital doctor" [56] — written by a recognized physician, married to a New York Times journalist, who helped him to envision the direction and writing of the book, in his own words-, has come to the attention of many readers, including biomedical informaticians. This author suggests that the "digital doctor" may still be premature, although promising in the future, with plenty of new applications. As suggested in the book, the past and present of BMI includes many failures, as shown by his analysis of electronic health records - a story that sometimes reminds one more of the Titanic than of what EHRs have actually achieved, despite their problems - a substantial advance for research and clinical medicine - or his statement about "the junkyard of failed computerized diagnostic systems built in the 1970s and 1980s" - undervaluing all the interesting ideas and advances that those pioneering systems introduced. Incidentally, at least one more issue indirectly relates both this "digital doctor" book and the paper by Martin-Sanchez and Campos. Both documents fail to mention Pubmed, probably the leading achievement of the whole area of BMI, which has transformed medicine and education. Even though Pubmed, and many achievements of the NLM and early biomedical informaticians seem frequently taken for granted, they are at the center of most biomedical and clinical research and practice - and will surely continue to be so in the future. And, similarly, little emphasis is placed in their manuscript on the evaluations that all these new approaches will require. Careful, controlled evaluations are a major issue for the many clinically-focused apps and microdevices that already crowd the technological market. Concepts such as "self-quantification" and "n-of-1 trials", mentioned in the manuscript, surely open up promising paths for research, but they also pose open questions about entirely novel scientific methods that will be required, and their bioethics implications. 


\section{Adapting to technological shifts}

Is this avalanche of technologies for medicine new? I work in a BMI group embedded in a school of computer science. While we are focused on our area, many of our neighbor groups - dedicated to topics such as ontologies, quantum computing, wireless devices, security, software analysis, data and text mining, planning, monitoring, etc — have worked, at some time, on medical applications. Usually they change topics when their interest ends, grants finish or fashionable trends shift to other areas. Should we demand exclusivity? Should we follow them, since they do not follow us? Of course not. We, as biomedical informaticians, cannot run after the last fashionable topic, trend or app, and change everything, from main research directions to $\mathrm{PhD}$ programs, unless some real breakthrough appears in informatics. In 1993, we did decide to do so at my group - like many BMI groups — when the Web showed its possibilities. But no clever group can be shifting its whole focus every two or three years, chasing after the latest technology.

Regarding some mentions that the authors make about the BMI situation in a couple of countries they are familiar with-Spain and Australia-, are they representative of BMI from an international perspective? For the Spanish situation — which I know well-, mentioned in the manuscript, there are deeper and more complex factors at work behind what they describe, which should require an extended commentary "per se". For Australia, special local circumstances may also apply. In the case of the European Commission support in the ICT for Health area, it is not accurate that most or all funding was at some time dedicated to the Virtual Physiological Human. I have been myself involved in various large projects that dealt directly with typical BMI issues - interoperability, clinical trials, and EHRs. The latter were and are also the focus of different directions under the EC umbrella.

The very term "digital medicine", central to the manuscript and previously proposed elsewhere, may be becoming increasingly popular — although such a popularity might be partially generated by media and bloggers, rather than by objective scientific reports alone-, but it seems an inadequate term for describing new trends in BMI. The shift from analog to digital machines and information processing in animals and machines were two of the main foundations of cybernetics, dating back to the work of Wiener in the 1940s [57]. With his cybernetics group, he changed the entire way of thinking and working in physiology, moving from the earlier focus on energy production and exchange to one emphasizing the coding, transmission, and feedback of information. Was this not already, in fact, the biological and social basis for digital medicine? And is it not also what we have been doing for decades in BMI? We all know that prospective biomedical informaticians may decide to forsake a research or academic path, when they realize that it is not necessary to follow a demanding $\mathrm{PhD}$ program in order to build a couple of apps that could make them millions very quickly, especially if they manage to sell their startups to Apple or Google. This fast lane existed long before Bill Gates decided to purchase a cheap operating system instead of studying computer science to learn how to write it himself, but trying to keep pace with technology cannot lead us to change $\mathrm{PhD}$ programs and their scientific foundations every few years. The separation between science and engineering in BMI has been often debated, and the best answer is that academic programs must teach students the foundations, methods and techniques of the area - both scientific and technological. Assuming such a premise, BMI programs can adapt to new technological advances or choose concrete paths — such as emphasizing data science, for instance, as is actually happening in some academic settings-, before major shifts in technology can lead old programs and people reluctant to change towards obsolescence. But this is what has always happened in all technological fields. 


\section{Scientific directions}

A quick search of the 20 top-cited medical informatics papers in the $\odot$ Thomson-Reuters' ISI Web of Science [58] - the fields of medical informatics and computational biology are separated in ISIshows that a good part of them have not really contributed to substantially advance the scientific basis of the discipline. Then, to follow the paths proposed by popular journal references - even with large numbers of citations - and even more media (particularly social media) seems dangerous or even frequently inadequate for scientific purposes. To cope with the needs of industry, academic programs must be aware of the specific knowledge that is required to train a good professional, but these programs cannot shift their own content to automatically adapt to any new fashionable trend. The number of directions that appeared at some point as "definitive" future directions in informatics —and later faded away - is quite large. If professionals from other academic disciplines or industry do not seek advice from BMI professionals we, biomedical informaticians, must consider what might be wrong, of course, but the solution is not to change the name of the field or follow these external professionals along every pathway they choose to take.

I will reuse here a proposal that I made at the recent MIE 2015, in a debate about the role of BMI today, led by Dr Reinhold Haux, former MIM editor. In 1903, Russell and Whitehead wrote the "Principia Mathematica" [59], where they aimed to synthesize the foundations of what was known then about mathematics. Earlier, in 1900, David Hilbert, the leading mathematician in Europe in a time of great competition, proposed twenty three fundamental problems in mathematics, unsolved at the time, which ended up defining the entire agenda of mathematics for decades [60]. Their solution led, for instance, to the establishment of some of the theoretical foundations of computer science. Some people have tried to do something similar in other fields but there has not been, to my knowledge, a similar effort carried out in BMI by one or two persons - rather than by a consensus group, where all the really challenging ideas that could lead, for instance, to a Nobel Prize, might be immediately discarded as impossible or too risky. I will mention here one possible set of topics, usually left outside BMI: studies of how information is coded and transmitted in the brain. Such discoveries might lead to fundamental changes in both science and medicine, but have never been, to my knowledge, a central issue for BMI. One can ask: why not?

\section{Conclusion}

The paper by Martin-Sanchez and Campos, while surely necessary and interesting, wanders among all these (apparently) new concepts without reaching a challenging conclusion. The authors present an interesting analysis of many of the terms and ideas around digital medicine, but one feels that something else is still needed to study the implications for BMI of the many issues surrounding digital medicine. Who could disagree — now or even a century ago — with the authors, when they state in the Conclusions section that "Medicine is now at a crossroad", that "From where we stand now, the challenges we face are endless" or that "There are no shortcuts on the road to success?" At the end, the paper lacks a concrete, practical thesis and specific recommendations that can serve as guides for future directions or actions. However, hopefully, the extended debate presented in this issue of the MIM journal will lead to new ideas for such an interesting topic. Besides that, the definitions and analysis of such a large number of terms and initiatives and its relationship with current views of BMI will surely be of great interest for professionals and students.

Let me finish with a digression about my favorite hobby, music. Today we have great chemical devices to detect and analyze any nanomolecule dissolved in a large pool, and the best sound analyzers 
of all times, but the techniques that Antonio Stradivari developed to build violins in the XVII-XVIII centuries or those for singing by Manuel Garcia - the best of all historic operatic teachers, who lived in the XIX century-, to train various generations of operatic singers, are mostly forgotten in their practical details. So, many specialists believe that we have worse violins and operatic singers than in the past centuries, in spite of all the available technologies in both areas. Hopefully, technologies will not advance so quickly that the next generations of biomedical informaticians will forget the lessons learned in more than half a century of BMI research while they rush to build new apps for the latest smartphones, connected to a myriad of external sensors and sources. 


\section{Comment by H.-A. Park}

Healthcare is facing a global crisis, due to increasing demand, increasing costs, decreasing reimbursements, shortages of healthcare workers, and misalignment of incentives.

Emerging information technologies such as EHR, advanced data analytics, mobile technologies, remote monitoring, wearable sensors, voice recognition, and new user interfaces can play an important role in solving these problems. However, most of these Health IT solutions are designed to improve efficiency, overlooking the patient who is a critical stakeholder in healthcare.

Marin-Sanchez and Lopez-Campos [1] emphasized the importance of the patient in healthcare by introducing participatory medicine as one of the two main areas of application for biomedical informatics research. Participatory medicine focuses on allowing patients to take control of their own health. Tom Ferguson first used the term e-patient in 1996 to describe a medical consumer who is "equipped, empowered and engaged" in his or her own healthcare [61]. Ferguson viewed the evolution of e-patients as part of a "paradigm shift" that is happening in medical care, from viewing laypeople as consumers of healthcare to seeing them as the primary providers of healthcare [62].

Health information technology is creating new opportunities for patients to be fully equipped, empowered, and engaged in their health management. I would like to discuss how patient empowerment and engagement can be facilitated using virtual agents and avatars in healthcare. Virtual agents and avatars are interactive characters that exhibit human-like qualities. A dramatic shortage of healthcare professionals, knowledge explosion in healthcare, ubiquity of smartphones across all socioeconomic groups, and increasing sophistication of artificial intelligence have all certainly contributed to the deployment of virtual health agents and avatars in healthcare.

I would like to introduce the virtual agents and avatars developed as part of a Korean Health Avatar project, in which I am involved as one of the co-investigators. In this project, a person ' $\mathrm{s}$ avatar is conceptualized as all health-related data about the person from genomic and clinical to lifelog data stored, accessed, and managed in a smartphone-enabled personal health record system supporting ASTM CCR (Continuity of Care Record) and HL7 CCD (Continuity of Care Document) standards for the IOS and Android mobile environment [63]. The avatar in this project is identical in concept to self-quantification -- which uses three types of data from the genome, the phenome, and the exposome -- as described in the MarinSanchez and Lopez-Campos article. Healthcare agents are conceptualized as an integrated and distributed intelligence program, which is expected to provide a variety of health services to the person avatars. Virtual agents can fill the gap by standing in as proxies for healthcare providers, and can be regarded as a subcomponent of what is referred to as precision medicine in the Marin-Sanchez and Lopez-Campos article. Individual person avatars interact with distributed healthcare agents for real-time personalized medicine through a platform, which also provides open APIs for the developers of distributed agents and profile registries for person avatars and healthcare agents. 
There are various types of virtual agents and avatars available through the platform. For example, there are virtual agents for health promotion, such as one used to encourage lifestyle changes for those with metabolic syndrome and obesity [64], one used to remind people of age-appropriate periodic health screening to promote early detection of diseases such as breast cancer, and one used to encourage and improve adherence to medication for those with hypertension and high cholesterol [65]. There are virtual agents and avatars for selfmanagement of chronic diseases such as Blue Avatar for the self-management of glucose, diet, exercise, lab, medication, and complications of diabetic patients. A virtual health agent that proactively reaches out to diabetic patients when they fail to enter their scheduled insulin injection can help patients to stay on track and prevent potentially precarious situations [66]. These types of virtual agents maximize patient engagement, particularly though personalized methods that employ motivational coaching, games, or social networking. Customized solutions to tackle specific conditions of patients, along with the ability to analyze personalized information, will create true wellness and self-management partners for patients.

There are virtual agents and avatars for predicting the risk of disease, or the probability of recovery or survival from a disease. For example, there are virtual agents predicting the occurrence of dementia, the probability of depression among adolescents, occurrence of adverse drug events, functional recovery from strokes, and survival from the top five cancers in Korea. There are virtual agents and avatars for disease management: for example. Avatar Beans representing chronic renal disease patients and DialysisNet representing health agents who take care of chronic renal patients, Avatar Jr. representing childhood cancer patients and KidsNet representing health agents who take care of childhood cancer patients, Avatar Gold representing colorectal patients and OncoNet representing health agents who take care of colorectal cancer patients. These types of virtual agents and avatars maximize the engagement of not only patients but also healthcare providers, particularly when employing evidencebased medicine.

While the use of virtual intelligent agent and avatar technology is still in the early stages in healthcare -- major social issues such as trust, security, and information privacy still remain to be navigated, and there are technical limitations regarding real-time perception, cognition, and action -- experts predict it will give patients and healthcare providers a new set of tools to better manage patients, increase patient empowerment and engagement, and improve patient adherence to treatment.

As Marin-Sanchez and Lopez-Campos concluded in their paper, no matter what approach we adopt from whichever direction, the most decisive factor of progress is the proper use of data, information, knowledge, and evidence for better healthcare. Again, this is where we, as biomedical informaticians, come in. 


\section{Comment by F. Sanz}

\section{An important field beyond nomenclature debates}

The stimulating paper of Martin-Sanchez and López-Campos [1] discusses from different relevant perspectives the current situation and the future prospects of the application of information technologies (IT) in the health field. They point out the overlap existing between the Digital Health and the Biomedical Informatics disciplines and analyze the popularity. Although I am not particularly interested in the debates about the nomenclatures used to refer to scientific disciplines, I think that this particular one reflects the existence of an emerging field that is changing the way in which health care and biomedical research are being carried out. In this context and following the reasoning line of the authors, I would like to stress the relation of the aforementioned disciplines with Bioinformatics and Computational Biology. Although these last disciplines are focused on the application of IT and computational methods to the analysis and understanding of biological data, most of them at the omics level and not necessary restricted to the biomedical field, they have today a clear focus on health related topics, and they are extending the scope of the considered data, including phenotypic and clinical ones. The European Genome-phenome Archive (EGA) constitutes a clear example of this evolution within the bioinformatics scientific community [67].

\section{The European Commission supports the medical application of bioinformatics}

The medical application of bioinformatics has been detected as a priority topic by the ongoing H2020 Program of the European Union, which launched a specific call for projects on the matter (PHC-32-2014. Advancing bioinformatics to meet biomedical and clinical needs). This call granted eight international projects, one of them being MedBioinformatics [68], which aims at developing integrative bioinformatics tools and software applications for analyzing the huge amount of data and knowledge generated in healthcare and biomedical research in order to facilitate translational research and precision medicine. A critical aspect that this project has detected is the need of an intense and active involvement of the intended final users (translational researchers and clinical practitioners) in the design and development of the applications. MedBioinformatics has been recently involved in the organization of the $1^{\text {st }}$ European Conference on Translational Bioinformatics held in Copenhagen on April 26-27 ${ }^{\text {th }}$, 2017, in parallel with similar initiatives existing in USA and Asia.

\section{Effective integration of different types of information (the Biomedical Big Data)}

A key challenge for advancing in Digital Health is the effective integration of heterogeneous (and incomplete) information of different types and from different sources, since the current existence of information silos and the insufficient availability of interoperability solutions are hampering the development of effective applications [69]. The types of information to be integrated includes clinical information, mainly from electronic health records, all the scope if high-throughput (omics) data, including information on how drugs and other chemicals 
interact with the proteome, and information automatically extracted from biomedical literature by text-mining. The DisGeNET database [70], a resource on gene-disease associations constitutes a clear example of these integrative efforts [71]. The same challenges and opportunities regarding holistic approaches are also applicable in the field of pharmaceutical research [72]. In this respect the European EU-ADR initiative in the field of pharmacovigilance demonstrated the power of the integrative approaches in pharmacovigilance by detecting 'prime suspects' and providing plausible biological mechanisms for them [73].

In summary, Digital Health-Biomedical Informatics-Bioinformatics is an exciting area that is already yielding significant results and that offers stimulating opportunities for contributing to scientific research, business development and citizens' health. 


\section{Comment by I. N. Sarkar}

As with any discipline, there are perennial concerns of whether and when the suggestion of a new discipline is complementary or competitive to biomedical informatics. Historically, biomedical informatics has seen this challenge time and time again. Perhaps most famously is the relationship between "bioinformatics" and "biomedical informatics." [49,50, 74-76] Of greater concern is when seemingly new disciplines emerge and gain rapid popularity without reference to the more than 60 years of relevant advances in biomedical informatics research and practice, such as noted by MartinSanchez and Lopez-Campos [1] with respect to digital health.

While the fundamental goal remains the same, to utilize data for advancing the understanding of the basis of disease and supporting health care, the labels used to refer to biomedical informatics have evolved through the years. This has undoubtedly led to confusion for the greater biomedical research community - are we experts in "medical informatics," "clinical informatics," "bioinformatics," or "biomedical and health informatics"? For most biomedical researchers it is not fathomable that a single discipline or individual can have deep expertise in more than one area of science. However, biomedical informatics is a macroscopic discipline, requiring a transdisciplinary approach to study health and support the practice of health care $[42,77]$. The biomedical informatics community continues to grapple with definitions and give rise to new labels, such as "translational bioinformatics" $[78,79]$ and "clinical research informatics." [80,81] Such introspection and precision in label making is valuable in contextualizing the application areas of biomedical informatics as well as providing the necessary plasticity in the discipline to accommodate advances in related disciplines, such as computer science, information science, and cognitive science.

The democratization of health data alongside near ubiquitous availability of computing resources has given rise to a new type of challenge for recognizing biomedical informatics contributions. As noted by Martin-Sanchez and Lopez-Campos, the increased profile of digital health in the last 15 years has been achieved with little to no direct mention or partnership with biomedical informatics. This is certainly troubling, but not entirely unexpected as one might perceive biomedical informatics as a conservative academic discipline, especially in light of our philosophical discussions about who we are and what we do [82-85]. The irony of this perception, of course, is that biomedical informatics, is a pragmatic science at its core aiming to make best use of available data from multiple sources for providing the best health care at the precisely correct time.

Amidst the significant advances that are poised to transform health care, a major shortfall of the biomedical informatics community has been its ability in describing to the broader scientific community what we do, how we do it, and our accomplishments (beyond electronic health records). The result, as further highlighted by Martin-Sanchez and Lopez-Campos, is the risk of competing educational and research initiatives in digital health, biomedical data science, health IT, etc. Even with the best intentions, relying on policy makers or administrators to provide the necessary administrative framework for allowing biomedical informatics to best address the acute challenge of best leveraging data for supporting health has resulted in awkward organizations (e.g., "biomedical informatics" divisions within "biomedical data science" departments, which is akin to putting a "biological sciences" division within a "microbiology" department) and coarse labeling of biomedical informatics with catchy terms like "eHealth" or "in silico medicine."

It is important to understand the nidus for digital health. Digital health is built on the foundation of [86]: (1) Rapid technological advances in computing and communication technologies; and (2) Understanding of data indicative of health state and disease risk, including use of molecular sequence as well as general fitness data. In contrast to the majority of research in biomedical informatics, where data are gathered by a health care providers and biomedical researchers, digital health is based on 
patient gathered data. Digital health is thus emblematic of patient empowered health care and therefore in stark contrast to traditional, provider-driven health care contexts. This, therefore suggests that digital health offers a new, technology-driven approach for next-generation health care [87]. It also acknowledges the current challenges in how health care is currently delivered, the cost inefficiencies in provider-driven diagnoses, and the general under utilization of available health data [88]. Within the realm of biomedical informatics, the sub-discipline of "consumer health informatics" (CHI) is most aligned with the axioms of digital health. There is thus a great opportunity for alliances between CHI working groups (e.g., the International Medical Informatics Association CHI Working Group) to be considered with colleagues in digital health.

The reflexive response to digital health for a biomedical informatics purist is perhaps to suggest that it is a mere distraction and, that it too will pass along with mHealth, health IT, or whatever phrase the media chooses to label our scientific contributions. However, there is a great opportunity to join the paradigm shift of health care delivery to more actively involve patients. As previously noted [89], by developing partnerships between $\mathrm{CHI}$ and the other facets of biomedical informatics, we can better approach health care challenges more holistically and identify the synergies with digital health. A major emphasis in recent years has been to leverage informatics approaches for supporting health care delivery, but there may be great merit in expanding to consider how one might support patients and preventative care. Indeed, many of the foundational methods developed and used in translational bioinformatics, clinical research informatics, and clinical informatics, just to name a few, might be combined with consumer health informatics methodologies to lead to a foundation that can catalyze digital health initiatives. Such synergies are in great demand by the patient community, and have resulted in the establishment of major entities focused on funding research specifically requiring patient engagement (e.g., the Patient-Centered Outcomes Research Institute in the United States [90]). Because each facet of biomedical informatics should involve health care providers and patients, it might be considered that each major sub-discipline of biomedical informatics identifies its relationship to consumer health informatics. The result would be a foundation of methodologies that not only demonstrate the relevance of biomedical informatics with respect to digital health, but also better support our common beneficiary: the patient. 


\section{Comment by H. Tanaka}

1. Introduction

In this issue, Martin-Sanchez et al. [1] wrote an excellent review paper on the new role which Biomedical Informatics should play in the era of so called "Digital Medicine". In a lot of points raised in this paper, I mostly agree with the scope of the authors on the new emerging trends, caused by the "Big Data" revolution in biomedical and health fields. Furthermore, in connection to these drastic changes, I also share the same fear with the authors that, if biomedical informatics fails to significantly embrace with these emerging new disciplines, it would be "in grave danger of becoming irrelevant."

The authors, however, do not explicitly describe the several crucial points regarding the advent of these new trends in "Digital Health"*. They are (1) changes of the basic mission and concept which medical care is generally expected to achieve in our current society, as the background and purpose of these new trends in Digital Health, (2) the essential features of "genuine" biomedical Big Data revolution, especially its difference from the conventional large data informatics and (3) the paradigm shifts in biomedicine and healthcare which this Big Data revolution is bringing us.

In describing above, I would mention that our current expertise in biomedical informatics successfully utilized in previous studies might not be no more so useful in coping with the radically new fields in Digital Health, mainly brought by biomedical Big Data revolution. Hence, we should conduct a revolutionary change even to radically alternate the established academic framework of our biomedical informatics, in order to embrace the new trends in Digital Health.

2. The paradigm shift of the mission and basic concept of medicine and healthcare in our present society.

What we should first take note of as a start of discussion is the drastic change regarding the mission and concept of medicine and healthcare in our society, due to the rapid arrival of "longevity society", where most of the people is well expected to live to the age of 90. As frequently denoted, the disease prevalence structure of our society has been radically changed for these decades, especially in western developed countries. Although cure of acute diseases in the hospital is still an important part of the medical practice, socially more important role is care of the age-related chronic diseases such as hypertension, diabetes and so forth, now spending more than half of the national health expenditures in many developed countries.

The age-related chronic disease is long-termed disease generally not cured completely. So, its medical care would be life-long, taken mainly out of hospitals. The concept of life-long prevention becomes extremely important for the age-related chronic disease, not only against onset of the disease (the primary prevention), but also, and more important, against recurrence, aggravation and complication of the disease (the secondary and tertiary 
prevention): for example, diabetes, if it is left untreated, causes the various complication such as renal failure requiring dialysis treatment which imposes a financial burden to national medical care expenditures.

In the medicine of previous era, as diseases to cure is mostly acute, medical care has no chance but to be "occasional" and "reactive" because most of the acute disease occurs abruptly, but in the era when the age-related chronic diseases are dominated, a concept of long-termed care through the life span, or longitudinal view of disease process become central, so that medical care is shifting to be much more "life-course-oriented" and "proactive" to the disease process.

In conclusion, the paradigm regarding the mission and concept of medicine and healthcare in current society is shifting from occasional and reactive medicine to "life-course-oriented proactive medicine". In other words, predictive, preventive [91] and preemptive medicine [92] are expected to be realized to satisfy needs of our "longevity society". Various new trends of Digital Health such as precision medicine and participatory healthcare are brought about in order to accomplish the life-course-oriented proactive medicine and healthcare.

3. The essential features of "genuine" biomedical Big Data and paradigm shifts Big Data revolution brought about

What we should take note of next is to understand the essential features of genuine "Big Data" revolution in biomedical and health fields. Recently, owing to the advances in biotechnology, vast amounts of biomedical data can be obtained with enormously reduced cost, but nevertheless with very high quality. Taking the human genome sequence as an example, time for reading the whole human genome is reduced about 10 thousand times less and its cost is also reduced more than 3 million times lower, during the recent 10 years, which exceeds by far Moore's law.

\subsection{Classification of the type of biomedical "Big Data"}

In large, there are two types of biomedical and health "Big Data". Difference between these two types of Big Data is crucial to understand the meaning of Big Data revolution.

\subsubsection{Conventional biomedical large amount of data}

One type is medical "Big Data" of "conventional" type, which collects the conventional medical information like clinical patient data (symptoms, laboratory tests, drug prescriptions, medical images etc.) or the epidemiological data (health survey or social inquiry about diet and life style etc.). On account of the rapid spread of digitalization of these conventional data, 
for example, by the nation-wide spread of, EHR, we now have a large digital repository of conventional clinical or epidemiological data such as nation-wide EHR databases in several countries.

This type of large conventional data, though often called "Big Data" wrongly, is nothing new, from information science, except that number of observed subjects (patients or participants) is "big", so that we will not call it "Big Data" hereafter in this comment. To gather a large number of subjects' data, however, might produce a new knowledge, which is not obtained by just seeing individual data alone, but emerges when large number of samples is collected, which is the long known principle called "law of large number" in statistics.

\subsection{2 "Genuine” biomedical Big Data}

Another type of Big Data is brand new and "genuine" biomedical Big Data. There are two subtypes of this genuine Big Data in biomedicine.

\section{(a) Genomic Big Data}

One subtype is comprehensive molecular information generally called "genomic Big Data" or "multi-omics Big Data". The clinical use of the genomic Big Data is already implemented in the cutting-edge hospitals [93], by sequencing patient's genome on their own, mainly (1) to identify the disease causative genetic aberration for undiagnosed and inherited disease at the point of care (POC) in clinical routine practice, or (2) to identify the driver mutation of cancer genes in order to assign the most appropriate molecularly-targeted anticancer drug to the patient.

Another fields utilizing genomic Big Data is genomic cohort/biobank, where genomes or omics of cohort participants are measured. The results are used, (1) in the population-based prospective genomic cohort (the cohort of healthy participants at least at the beginning of follow-up), to predict the relative risk of participants for onset of various diseases, and, (2) in the disease-oriented genomic cohort (cohort of the patients contracting specific diseases), to investigate the correlation between patient's molecular information and clinical phenotypes such as disease outcome, providing the basic information to realize the personalized medicine. Thus, the biomolecular Big Data of genomic cohort is expected to provide an information basis to be utilized for realization of genome-omics medicine or healthcare.

The genomic or multi-omics Big Data are usually measured "in hospital" setting but recently measurement of these data can be executed in daily life setting. This kind of measurement is called "liquid biopsy" which observes the circulating tumor cells (CTC), circulating cell free DNA (ctDNA), extracellular RNA (exRNA), microRNA (miRNA), and exosome (cell-derived vesicle) that would be good biomarker for early diagnosis of cancerous, inflammatory, cardiovascular and metabolic diseases.

(b) mHealth Big Data 
Another subtype of the genuine Big Data in healthcare is mobile health (mHealth) Big Data. On account of the rapid progress in sensor technology such as flexible organic electronics and smart IT revolution, unobtrusive and "continuous" monitoring of vital signals and behaviors by wearable sensors can be executed in natural settings. Each set of observed data is not so big, but accumulation of " 24 hours continuous measurement" produces a mHealth Big Data, which develops new metrics and more effective interventions into health conditions (physical activity, sleep, depression, cognition, diet, etc.) with the use of smart data communication and processing devices.

\subsection{Crucial difference between genuine Big Data and conventional large amounts of data}

We should recognize the essential features of the "genuine" biomedical Big Data.

(a) Crucial difference in the purpose to collect Big Data

First, the purpose to collect genuine Big Data is quite different from that of collecting conventional large amounts of data. As I mentioned before, the purpose of conventional large amount of data is to reveal the hidden order or law which is not visible by observing small number of samples but emerges only when a sufficient number of samples are gathered. But the purpose of "genuine biomedical Big Data" is quite different from this collective approach.

Recent advances in clinical genome and multi-omics medicine have clarified that even if the disease name is same, there are variety of "intrinsic subtypes" due to the difference of underlying genomic and omics mechanism of disease which necessitates the "personalized medical treatment" for individual patient. Hence, the purpose for data collection in the genuine biomedical Big Data is to observe how many distinct "personalized subtypes" or "stratification" of disease are existing, to make the comprehensive "personalized medicine". In the case of population-based cohort, how many personalized subtypes of disease occurring mechanism are existing, to make the "personalized prevention" complete. Hence we should collect as many as subjects (patients or participants) to exhaust all the personalized subtypes. The direction of the study is toward personalization not a collective law.

(b) "small $n$ big $p$ problem" and need for radically new informatics for Big Data

The second important feature is that, in contrast to the conventional medical large data where only number of subjects $(n)$ is "big", in the genuine biomedical Big Data, number of observed items or attributes $(p)$ are extraordinarily "Big". For example, sequencing the human genome produces three billion nucleotide data and if we measure other omics, the resultant number of measured data variables becomes so enormous. This situation is also valid for mHealth Big Data, continuous measurement of vital and behavioral signals.

Comparing the number of measured items $(p)$, the number of subjects (patients or cohort participants) are, though effort is made to collect as many subjects as possible (for example, a half million participants in UK biobank [94]), several order of magnitude smaller than the number of attributes ( $\mathrm{p}>>\mathrm{n})$, which is called "small $n$ big $p$ problem" [95] in statistics which makes most of statistical methods (especially, multivariate analysis) invalid or useless. Only 
the new informatics such as "sparse data modelling" [96] or "deep learning" [97] can be utilized as effective analytical tools.

3.3 Paradigm shifts in biomedicine and healthcare which Big Data revolution brought about _-_-From RCT to Real World Data

We have already mentioned several paradigm shifts which Big Data revolution has brought about in medicine and healthcare. The authors also wrote several paradigm shifts in their paper. The first paradigm shift to denote is that from population medicine ("one size fits for all” medicine) to personalized (stratified) medicine which genomic and multi-omics Big Data has brought by revealing the diversity of disease subtypes. The second would be that from reactive to proactive medicine, which is already described in detail in section 2 of this comment. As similar concepts to this shift, we have mentioned about the predictive, preventive and preemptive medicine.

There are several other paradigm shifts which the Big Data revolution has brought about such as participatory medicine or "exposomic" healthcare (including environmental factors' effect to health). As there is not sufficient space to discuss them, I only mention one of the important paradigm shift due to Big Data revolution which Sanchez et al.'s paper does not describe; the paradigm shift from randomized controlled trial (RCT) to real world data research, which means the RCT in clinical research.

From the concept of evidence-based medicine, RCT has been the "golden standard" for the clinical research. But, as it becomes widely known that there are so many personalized disease subtypes under the same entity of disease, it would be virtually impossible to make a randomized subject group for clinical trial which comprehensively subsumes all the personalized subtypes of disease, so that the group for RCT should be forced to be genetically biased. We have Big Data, which is full of real world data with number of subjects approaching to "population" in statistical meaning. We do not have to stick to the randomized "sampling" of subjects to make artificial clinical trial group, but directly use the real world data to extract the real world knowledge. Institute of Medicine (IOM) proposed the Learning Health System [98] which extracts the knowledge from real world clinical data while practicing medicine.

4. Our strategy to embrace the genuine Big Data revolution and Digital Health --- The third revolution of the medical informatics

As I mentioned, the importance of biomedical Big Data revolution is not that data is just big, but, more important, it is now causing the essential paradigm shifts of biomedical and health.

The biomedical Big Data revolution impose us, essentially new challenges in the biomedical informatics. In coping with them, we should not stick to conventional biomedical 
informatics method based on the past success experience in the clinical information system. The challenges are essentially new. Hence, we should abandon such an idea that we are expertized in handling the biomedical data, in order to be truly aggressive to cope with the brand new challenges which Digital Health has opened, such as to resolve "small-n, big-p problem" or reveal the reason why "deep learning" achieves a good performance for the biomedical problem, too.

Looking back at the history of medical informatics as academic discipline, there have been two revolutionary changes which even alternated the academic framework of medical informatics to subsume the new emerging fields.

The medical informatics started originally as digitalization or computerization of clinical information to establish the quantified objective medicine, a kind of similar concept as current "precision medicine". Especially digitalization of patient medical record (EMR) and statistical analysis of diagnostic/therapeutic process were pursuit at that time [99].

First revolutionary change has taken place at late 70 s or early 80 s, due to the advent of AI (artificial intelligence) in medical informatics. Concretely medical expert systems like MYCIN, Internist-I and CASNET, executing medical diagnosis and giving therapeutic advice to the physicians. As the medical informatics at that time is mainly statistics-based, knowledge-based system is really a huge impact to it. The community of medical informatics was, however, very positive to include not only knowledge-based medical decision support systems but also knowledge science by alternating the framework of medical informatics by placing the conceptual study of medical knowledge in general (medical ontology and terminology) at the foundation level of medical informatics.

The second revolution was occurred due to the advent of bioinformatics and genome omics medicine in late $90 \mathrm{~s}$ and early 00s. The rapid progress in genome omics science and its application to biomedicine have quaked the medical informatics community; at that time medical informatics only dealt with clinical phenotypic information. Although the academic basis (molecular genetics) to understand the new field such as bioinformatics or genome science has been unrelated to medical informatics community, it was very positive to include the bioinformatics or genome science, as long as these disciplines are related to clinical medicine. This subfield is now called "translational bioinformatics". Even by changing the name of the discipline from "medical informatics" to "biomedical informatics", our medical informatics community successfully subsumed the newly emerging disciplines, especially the American Medical Informatics Association (AMIA) was well taking in this new field.

Hence, I think this time advent of Big Data revolution and mobile or smart Digital Health would also demand the revolutionary change of our medical informatics society to cope with them. As in the two previous revolution, alternating the academic framework of biomedical informatics from its basis would be necessary. The revolutionary change this time would be to place the concept of life-course-oriented medicine and healthcare as the basis of our academic framework and new generation AI or machine learning method like deep learning as a central information tool for analyzing Big Health Data to extract new knowledge. 
We should be modest to throw away our pride based on our past success of biomedical informatics. We are not privileged, in comparison to new comers in the Digital Health. We should devote ourselves to cope with the new emerging fields without any preconceived notion. The biomedical informatics has already overcome such a trial two times. Thus, I am quite optimistic about getting through the trial of this time, too.

*(note): Hereafter we use this term with rather comprehensive meaning than "digital medicine". 


\section{References}

[1] Martin-Sanchez FJ, Lopez-Campos GH. The New Role of Biomedical Informatics in the Age of Digital Medicine. Methods Inf Med. 2016;55(?):xx-yy. -THIS ISSUE

\section{References Al-Shorbaji}

[2] Al-Shorbaji N. eHealth to go beyond data processing in response to public and individual health challenges. PAHO eHealth Newsletter, Vol. 2, No. 1 (January 2014). (http://www.paho.org/ict4health/newsletter/eHealth_Newsletter_January13.html)

[3] Scott RE, Mars M. A globally accepted e-health terminology? Presented at Med-e-Tel: The International eHealth, Telemedicine, and Health ICT Forum for Education, Networking and Business LuxExpo, Luxembourg, Luxembourg 20-22 April 2015 https://www.medetel.eu/download/2015/parallel_sessions/presentation/day3/Terminology.pdf

[4] Oh H, Rizo C, Enkin M, Jadad A. What is eHealth (3): A Systematic Review of Published Definitions. J Med Internet Res. 2005 Feb 24;7(1):e1

[5] Pagliari C, Sloan D, Gregor P, Sullivan F, Detmer D, Kahan JP, et al. What is eHealth (4): a scoping exercise to map the field. J Med Internet Res. 2005 Mar31; 7 (1): e9. (http://www.ncbi.nlm.nih.gov/pubmed/15829481)

[6] World Health Organization. WHA58.28 eHealth. http://apps.who.int/iris/bitstream/10665/20378/1/WHA58_28-en.pdf?ua=1

[7] Bioinformatics.

Oxford

Dictionaries

(http://www.oxforddictionaries.com/definition/english/bioinformatics)

[8] Hersh W. Who are the Informaticians? What We Know and Should Know. J Am Med Inform Assoc. 2006 Mar-Apr; 13(2): 166-70. doi: 10.1197/jamia.M1912 (http://www.ncbi.nlm.nih.gov/pmc/articles/PMC1447543/)

[9] Interdisciplinary. The Free Dictionary on the Internet. (http://encyclopedia.thefreedictionary.com/Interdisciplinarity

[10] Bioinformatics. The Free Dictionary on the Internet. (http://encyclopedia.thefreedictionary.com/Interdisciplinarity)

[11] Bahagon Y, Jacobson O. eHealth, mHealth and healthier social media reform: the big scale view. Int J Integr Care. 2012 Apr-Jun; 12(Suppl1): e106. (http://www.ncbi.nlm.nih.gov/pmc/articles/PMC3571141/)

[12] Van Velsen L, Wentzel J, Van Gemert-Pijnen JE. Designing eHealth that Matters via a Multidisciplinary Requirements Development Approach. JMIR Res Protoc. 2013 Jun 24;2(1):e21. doi: 10.2196/resprot.2547 (http://www.ncbi.nlm.nih.gov/pubmed/23796508)

[13] RIO+, United Nations Conference on Sustainable Development, Rio de Janeiro, Brazil, on June 20-22, 2012 (http://www.uncsd2012.org/) 
[14] United Nations General Assembly Resolution A/RES/70/1 (September 2015) http://www.un.org/ga/search/view_doc.asp?symbol=A/RES/70/1\&Lang=E

[15] Wild CP. Complementing the genome with an "exposome": the outstanding challenge of environmental exposure measurement in molecular epidemiology. Cancer Epidemiol Biomarkers Prev. 2005 Aug; 14 (8): 1847-50. http://cebp.aacrjournals.org/content/14/8/1847.

[16] World Health Organization. The World Health Report 2013: research for universal health coverage.- Geneva: World Health Organization, 2013. P. 20.

[17] World Health Organization. World Health report 2010: "Health systems financing: the path to universal coverage.- Geneva: WHO, 2010. (http://www.who.int/whr/2010/en/)

[18] United Nations General Assembly. Universal Health Coverage. 2 December 2012 (https://documents-dds-

ny.un.org/doc/UNDOC/LTD/N12/630/51/PDF/N1263051.pdf?OpenElement

\section{References Bellazzi}

[19] Bernstam EV, Smith JW, Johnson TR. What is biomedical informatics? J Biomed Inform. 2010 Feb;43(1):104-10.

[20] Coiera R. Guide to Health Informatics, CRC Press, 2015.

[21] Elenko E, Underwood L, Zohar D. Defining digital medicine. Nat Biotechnol. 2015 May;33(5):456-61.

[22] Ohno-Machado L. NIH's Big Data to Knowledge initiative and the advancement of biomedical informatics. J Am Med Inform Assoc. 2014 Mar-Apr;21(2):193.

[23] Lopez-Campos GH, Lopez-Alonso V, Martin-Sanchez F. Is Precision Medicine different from Personalised Medicine? A Biomedical informatics perspective. Stud Health Technol Inform. 2014;202:20-3.

[24] Chisholm RL. At the Interface between Medical Informatics and Personalized Medicine: The eMERGE Network Experience. Healthc Inform Res. 2013 Jun;19(2):67-8.

[25] Lopez-Campos G, Ofoghi B, Martin-Sanchez F. Enabling Self-Monitoring Data Exchange in Participatory Medicine. Stud Health Technol Inform. 2015;216:1102.

[26] Martin Sanchez F, Gray K, Bellazzi R, Lopez-Campos G. Exposome informatics: considerations for the design of future biomedical research information systems. J Am Med Inform Assoc. 2014 May-Jun;21(3):386-90.

[27] Ückert F, Ammenwerth E, Dujat C, Grant A, Haux R, Hein A, Hochlehnert A, KnaupGregori P, Kulikowski C, Mantas J, Maojo V, Marschollek M, Moura L, Plischke M, Röhrig R, Stausberg J, Takabayashi K, Winter A, Wolf KH, Hasman A. Past and next 10 years of medical informatics. J Med Syst. 2014 Jul;38(7):74. 
[28] Bellazzi R. Big data and biomedical informatics: a challenging opportunity. Yearb Med Inform. 2014 May 22;9:8-13.

[29] Williams MS, Ritchie MD, Payne PR. Interdisciplinary training to build an informatics workforce for precision medicine. Appl Transl Genom. 2015 Aug 4;6:28-30.

[30] Otero P, Hersh W, Jai Ganesh AU. Big Data: Are Biomedical and Health Informatics Training Programs Ready? Contribution of the IMIA Working Group for Health and Medical Informatics Education. Yearb Med Inform. 2014 Aug 15;9:177-81. doi: 10.15265/IY-20140007 .

[31] Kulikowski CA, Shortliffe EH, Currie LM, Elkin PL, Hunter LE, Johnson TR, Kalet IJ, Lenert LA, Musen MA, Ozbolt JG, Smith JW, Tarczy-Hornoch PZ, Williamson JJ. AMIA Board white paper: definition of biomedical informatics and specification of core competencies for graduate education in the discipline. J Am Med Inform Assoc. 2012 NovDec;19(6):931-8.

\section{References de Quiros}

[32] Collins FS, Varmus H. A new initiative on precision medicine. The New England journal of medicine. 2015 Feb 26;372(9):793-5.

[33] Jameson JL, Longo DL. Precision medicine--personalized, problematic, and promising. The New England journal of medicine. 2015 Jun 4;372(23):2229-34.

[34] Alyass A, Turcotte M, Meyre D. From big data analysis to personalized medicine for all: challenges and opportunities. BMC medical genomics. 2015;8:33.

[35] Topol EJ. The creative destruction of medicine : how the digital revolution will create better health care. 1st pbk. ed. New York: Basic Books; 2013. xi, 319 p. p.

[36] Lefebvre RC, Bornkessel AS. Digital social networks and health. Circulation. 2013 Apr 30;127(17):1829-36.

[37] Valente TW. Network interventions. Science. 2012 Jul 6;337(6090):49-53. 


\section{References Koch}

[38] Ortiz E, Clancy CM. Use of Information Technology to Improve the Quality of Health Care in the United States. Health Serv Res 2003;38(2):xi-xxii.

[39] Dolan B. Healthcare still lags behind other industries in leveraging mobile, CIO survey finds. MobileHealthNews. http://mobihealthnews.com/31404/healthcare-still-lags-behindother-industries-in-leveraging-mobile-cio-survey-finds (last accessed 2016-05-03).

[40] Eysenbach G. What is e-health? J Med Internet Res 2001;3(2):e20.

[41] Hersh W. A stimulus to define informatics and health information technology. BMC Med Inf Dec Mak 2009;9:24.

[42] Hasman A, Ammenwerth E, Dickhaus H, Knaup P, Lovis C, Mantas J, Maojo V, MartinSanchez FJ, Musen M, Patel VL, Surján G, Talmon JL, Sarkar IN. Biomedical Informatics A Confluence of Disciplines? Methods Inf Med 2011;50(6):508-524.

[43] Maojo V, Kulikowski CA. Note on Friedman's 'what informatics is and isn't'. J Am Med Inform Assoc 2013;20:e365-e366.

[44] Koch S, Vimarlund V. Critical Advances in Bridging Personal Health Informatics and Clinical Informatics. Yearb Med Inform 2012;7(1):48-55.

[45] Zakim D, Fritz C, Braun N, Fritz P, Alscher MD. Computerized history-taking as a tool to manage dyslipidemia. Vasc Health Risk Manag. 2010;6:1039-1045.

[46] PewResearchCenter. http://www.pewinternet.org/2013/01/28/tracking-for-health/. Last access: May 15, 2016.

[47]

http://quantifiedself.com/symposium/Symposium2014/QSPublicHealth2014_Report.pdf. Last access: May 15, 2016.

[48] Kravitz RL, Duan N, Vohra S, Li J, the DEcIDE Methods Center N-of-1 Guidance Panel. Introduction to N-of-1 Trials: Indications and Barriers. In: Kravitz RL, Duan N, eds, and the DEcIDE Methods Center N-of-1 Guidance Panel (Duan N, Eslick I, Gabler NB, Kaplan HC, Kravitz RL, Larson EB, Pace WD, Schmid CH, Sim I, Vohra S). Design and Implementation of N-of-1 Trials: A User's Guide. AHRQ Publication No. 13(14)-EHC122-EF. Rockville, MD: Agency for Healthcare Research and Quality; February 2014: Chapter 1, pp. 1-11. 


\section{References Kulikowski}

[49] Maojo V, Kulikowski CA. Medical Informatics and Bioinformatics: Integration or Evolution through Scientific Crises? Methods Inf Med. 2006; 45(5): 474-82.

[50] Kulikowski CA. The Micro-Macro Spectrum of Medical Informatics Challenges: From Molecular Medicine to Transforming Health Care in a Globalizing Society. Methods Inf Med. 2002; 41(1):20-4.

[51] Coiera E, Aarts J, Kulikowski C. The Dangerous Decade. J Am Med Inform Assoc. 2012 Jan-Feb; 19(1):2-5..

\section{References Lovell}

[52] 'A simple explanation of the Internet of Things', http://www.forbes.com/sites/jacobmorgan/2014/05/13/simple-explanation-internet-thingsthat-anyone-can-understand accessed 18/4/2016.

[53] ' $\$ 117$ Billion Market For Internet of Things In Healthcare By 2020', http://www.forbes.com/sites/tjmccue/2015/04/22/117-billion-market-for-internet-of-things-inhealthcare-by-2020 accessed 18/4/2016.

[54] Zhang YT, Poon CC. Editorial note on bio, medical, and health informatics. IEEE Transactions on Information Technology in Biomedicine. 2010;14(3):543-5.

[55] 'How to avoid FDA regulation of your mobile medical app', http://www.meddeviceonline.com/doc/how-to-avoid-fda-regulation-of-your-mobile-medicalapp-0001 accessed 22/4/2016.

\section{References Maojo}

[56] The Digital Doctor: Hope, Hype, and Harm at the Dawn of Medicine's Computer Age. McGraw-Hill. April, 2015

[57] Wiener N. Cybernetics: Or Control and Communication in the Animal and the Machine. The MIT Press 1948, 2nd edition. 1961.

[58] http://ipscience.thomsonreuters.com/product/web-of-science/

[59] Whitehead AN, Russell B. Principia Mathematica (1st ed.), Cambridge: Cambridge University Press. 1910.

[60] http://aleph0.clarku.edu/ djoyce/hilbert/problems.html 


\section{References Park}

[61] Levin LS, Idler EI. The Hidden Health Care System: Mediating Structures and Medicine. . Cambridge, Massachusetts, Ballinger Publishing Co. 1981.

[62] Ferguson T. e-Patients: How They Can Help Us Heal Health Care 2007, Available from: http://e-patients.net/e-Patients_White_Paper.pdf

[63] Kim JH. Health Avatar: An Informatics Platform for Personal and Private Big Data. Heal thc Inform Res. 2014 Jan; 20(1): 1-2. doi: 10.4258/hir.2014.20.1.1

[64] Jeon E, Park H-A. Development of a Smartphone Application for Clinical-Guideline-Bas ed Obesity Management. Healthc Inform Res. 2015; 21(1): 10-20. doi: 10.4258/hir.2015.21. 1.10

[65] Kang H, Park H-A. A Mobile App for Hypertension Management Based on Clinical Prac tice Guidelines: Development and Deployment. JMIR Mhealth Uhealth. 2016; 4(1):e12. doi: 10.2196/mhealth.4966.

[66] Jo S, Park H-A. Development and Evaluation of a Smartphone Application for Managing Gestational Diabetes Mellitus. Healthc Inform Res. 2016; 22(1):11-21. http://dx.doi.org/10.4 258/hir.2016.22.1.11 


\section{References Sanz}

[67] Lappalainen I, Almeida-King J, Kumanduri V, Senf A, Spalding JD, Ur-Rehman S, et al. The European Genome-phenome Archive of human data consented for biomedical research. Nat Genet. 2015;47(7):692-5.

[68] http://www.medbioinformatics.eu/. Accessed on May $24^{\text {th }}, 2016$.

[69] Cases M, Furlong LI, Albanell J, Altman RB, Bellazzi R, Boyer S, et al. Improving data and knowledge management to better integrate health care and research. J Intern Med. 2013;274(4):321-8.

[70] http://www.disgenet.org/ . Accessed on May 24 ${ }^{\text {th }}, 2016$.

[71] Queralt-Rosinach N, Piñero J, Bravo À, Sanz F, Furlong LI. DisGeNET-RDF:

harnessing the innovative power of the Semantic Web to explore the genetic basis of diseases. Bioinformatics. 2016 Apr 22. [Epub ahead of print].

[72] Marti-Solano M, Birney E, Bril A, Della Pasqua O, Kitano H, Mons B, et al. Integrative knowledge management to enhance pharmaceutical R\&D. Nat Rev Drug Discov. 2014;13(4):239-40.

[73] Coloma PM, Schuemie MJ, Trifirò G, Furlong L, van Mulligen E, Bauer-Mehren A, et al. EU-ADR consortium. Drug-induced acute myocardial infarction: identifying 'prime suspects' from electronic healthcare records-based surveillance system. PLoS One. 2013;8(8):e72148.

\section{References Sarkar}

[74] Maojo V, Kulikowski CA. Bioinformatics and medical informatics: collaborations on the road to genomic medicine? J Am Med Inform Assoc. 2003;10(6):515-22.

[75] Martin-Sanchez F, Iakovidis I, Norager S, Maojo V, de Groen P, Van der Lei J, et al. Synergy between medical informatics and bioinformatics: facilitating genomic medicine for future health care. J Biomed Inform. 2004;37(1):30-42.

[76] Maojo V, Martin-Sanchez F, Billhardt H, Iakovidis I, Kulikowski C. Establishing an agenda for biomedical informatics. Methods Inf Med. 2003;42(2):121-5.

[77] Sarkar IN. Biomedical informatics and translational medicine. J Transl Med. 2010;8:22.

[78] Butte AJ. Translational bioinformatics: coming of age. J Am Med Inform Assoc. 2008;15(6):709-14.

[79] Sarkar IN, Butte AJ, Lussier YA, Tarczy-Hornoch P, Ohno-Machado L. Translational bioinformatics: linking knowledge across biological and clinical realms. J Am Med Inform Assoc. 2011;18(4):354-7.

[80] Embi PJ, Payne PR. Clinical research informatics: challenges, opportunities and definition for an emerging domain. J Am Med Inform Assoc. 2009;16(3):316-27. 
[81] Embi PJ, Payne PR, Kaufman SE, Logan JR, Barr CE. Identifying challenges and opportunities in clinical research informatics: analysis of a facilitated discussion at the 2006 AMIA Annual Symposium. AMIA Annu Symp Proc. 2007:221-5.

[82] Bernstam EV, Smith JW, Johnson TR. What is biomedical informatics? J Biomed Inform. 2010;43(1):104-10.

[83] Friedman CP, Ozbolt JG, Masys DR, American College of Medical I. Toward a new culture for biomedical informatics: report of the 2001 ACMI symposium. J Am Med Inform Assoc. 2001;8(6):519-26.

[84] Kahn MG. The expanding informatics community: blessing or curse? J Am Med Inform Assoc. 2001;8(6):621-3.

[85] Moore JH, Holmes JH. The golden era of biomedical informatics has begun. BioData Min. 2016;9:15.

[86] Topol EJ. The creative destruction of medicine : how the digital revolution will create better health care. New York: Basic Books; 2012. xi, 303 p. p.

[87] Elenko E, Underwood L, Zohar D. Defining digital medicine. Nat Biotechnol. 2015;33(5):456-61.

[88] Steinberg D, Horwitz G, Zohar D. Building a business model in digital medicine. Nat Biotechnol. 2015;33(9):910-20.

[89] McDaniel AM, Schutte DL, Keller LO. Consumer health informatics: from genomics to population health. Nurs Outlook. 2008;56(5):216-23 e3.

[90] Selby JV, Beal AC, Frank L. The Patient-Centered Outcomes Research Institute (PCORI) national priorities for research and initial research agenda. JAMA. 2012;307(15):1583-4.

\section{References Tanaka}

[91] Hood L, Flore M. A personal view on systems medicine and the emergence of proactive P4 medicine: predictive, preventive, personalized and participatory.N Biotechnol. 2012 Sep 15; 29(6):613-24. doi: 10.1016/j.nbt.2012.03.004. Epub 2012 Mar 18.

[92] Zerhouni E. Strategic vision for the future: from curative to preemptive medicineNational Institutes of Health, 2008

[93] Worthey EA, Mayer AN, Syverson GD, Helbing D, Bonacci BB, Decker B, et. al., Making a definitive diagnosis: successful clinical application of whole exome sequencing in a child with intractable inflammatory bowel disease.Genet Med. 2011 Mar; 13(3):255-62.

[94] Collins R. What makes UK Biobank special? Lancet 2012; 379(9822): 1173-4.

doi:10.1016/S0140-6736(12)60404-8 
[95] Zinovyev A. Overcoming Complexity of Biological Systems: from Data Analysis to Modelling. Math. Model. Nat. Phenom. 2015;10(3): 186-205.

[96] Xu Z, Shi Y.Exploring Big Data Analysis: Fundamental Scientific Problems. Annals of Data Science, 2015; 2(4):363-367.

[97] Hinton GE, Osindero SA, The YW. Fast Learning Algorithm for Deep Belief Nets. Neural Comput. 2006 Jul; 18(7):1527-1554.

[98] Institute of Medicine (US) Roundtable on Evidence-Based Medicine; Aisner D, Olsen LA, McGinnis JM, editors. The Learning Healthcare System: Workshop Summary.Washington (DC): National Academies Press (US); 2007. The National Academies Collection: Reports funded by National Institutes of Health.

[99] Shortliffe T, Cimino J (eds). Biomedical Informatics - Computer Applications in Health Care and Biomedicine (4th edition), Springer 2013.

\section{Addresses of the authors:}

Najeeb Al-Shorbaji

E-mail: shorbajin@gmail.com

Riccardo Bellazzi

Dipartimento di Ingegneria Industriale e dell'Informazione Università degli Studi di Pavia

Via Ferrata 3

27100 Pavia

Italy

E-mail: riccardo.bellazzi@ unipv.it

Fernan G. B. de Quiros

Fernan Gonzalez Bernaldo de Quiros

Direccion Medica

Hospital Italiano de Buenos Aires

Peron 4190

(1199) Ciudad Autonoma de Buenos Aires

Argentina

E-mail: fernan.quiros@ hospitalitaliano.org.ar

Sabine Koch

Health Informatics Centre

LIME

Karolinska Institutet

SE 17177 Uppsala

Sweden 
E-mail: sabine.koch@ki.se

Casimir A. Kulikowski

The State University of New Jersey Rutgers

Department of Computer Science

Hill Center, Busch Campus

New Brunswick, New Jersey 08903

USA

E-mail: kulikows@cs.rutgers.edu

Nigel Lovell

Faculty of Engineering

UNSW

Samuels Building, Lower Ground

Sydney NSW 2052

Australia

E-Mail: n.lovell@unsw.edu.au

Victor Maojo

Departamento de Inteligencia Artificial

Facultad de Informática

Universidad Politécnica de Madrid

Campus de Montegancedo s/n

28660 - Boadilla del Monte (Madrid)

Spain

email: vmaojo@infomed.dia.fi.upm.es

Hyeoun-Ae Park

College of Nursing \& Systems Biomedical Informatics Research Center

Seoul National University

28 Yongon-dong, Chongno-gu

Seoul 110-799

Korea

E-Mail: hapark@snu.ac.kr

Ferran_Sanz

Molecular Informatics Research Group

Department of Experimental and Health Sciences

Universitat Pompeu Fabra

Barcelona

Spain

E-mail: ferran.sanz@upf.edu 
I. Neil Sarkar

Brown University

Center for Biomedical Informatics

Providence

Rhode Island 02912

USA

E-mail: neil_sarkar@brown.edu

Hiroshi Tanaka

University Center for Information Medicine

Tokyo Medical and Dental University

Medical Research Institute

1-5-45 Yushima, Bunkyo

Tokyo 113-8510

Japan

E-mail: tanaka@cim.tmd.ac.jp 